\title{
Towards chemical mapping at sub-micron resolution: near-field spectroscopic delineation of interphase boundaries
}

\author{
Hubert Pollock \\ Department of Physics, University of Lancaster, Lancaster LA1 4YB, England \\ h.m-pollock@lancaster.ac.uk
}

Keywords: microspectroscopy, sub-micro, PTMS, PTIR, PCA-LDA, fuzzy rule, eClas, biomarkers, adult stem cell

\begin{abstract}
Several of the authors of this collection of papers presented at the international meeting on the mechanical behaviour of materials have been working continuously in that field for several decades. In contrast, in this instance we have an example of an author who, having some experience in nanoindentation and surface - mechanical research, now pursues interdisciplinary studies of nanoscale properties in a different field.

This paper discusses how a near-field version of infrared microspectroscopy, together with multivariate data analysis points a way towards a new method for identifying biomarkers for use in biomedical evaluation procedures. We also outline some details of a non-statistical method of classification, employing fuzzy logic.
\end{abstract}

\section{Introduction}

In a wide range of situations ranging from polymer technology to biomedical studies, the samples to be characterised can be inhomogeneous, with structural detail at the sub-micrometer level. The researcher often faces the task of chemically mapping the individual components or detecting subtle spatial variations in their molecular composition. Hence our interest in the microspectroscopical properties of materials. We discuss two examples of recent work carried out in collaboration with colleagues at the universities of Lancaster and East Anglia, who have employed combinations of scanning probe microscopy with infrared spectroscopy for chemical mapping by infrared microspectroscopy at sub-micron spatial resolution. Accordingly, this paper, as presented to a meeting on the mechanical behaviour of materials, is in the nature of a non-specialist review. Biomedical specialists will find that statements and claims made here are justified by reference to previously published papers, as indicated in the text.

\section{Near-field spectroscopic characterization of biomaterials}

Localised spectroscopic techniques. The first example is taken from the biomedical field, where the motive was to identify biochemical markers that might serve as diagnostic fingerprints for identifying rogue cells of human tissue. These markers would consist of certain features of vibrational spectra in the mid-infrared, enabling us to pick out molecular groups of interest. The expectation was that any spectral deviations would point to the presence of biomolecular alterations within cells. If, for example, an automated procedure could thereby be found that might replace the visual procedures involved in screening, in the UK, four million women per year for cervical cancer, there would in principle be good prospects of very significantly reducing the proportion of false negatives and false positives. Typically the proportion of suspicious cells in a sample may be less than $2 \%$, and even existing semi-automated morphometric and related techniques designed to address such issues have yet to prove themselves more cost-effective and reliable than conventional labour-intensive approaches.

The task of obtaining and interpreting localised spectral data from individual cells or groups of cells, requiring the development of a combination of spectroscopy with scanning probe microscopy, involved expertise in several areas: near-field microscopy, evanescent thermal waves, the photothermal effect, multivariate data analysis, spectroscopic analysis... a truly interdisciplinary 
undertaking. Infrared microspectroscopy has already reached the point at which appropriate methods of analysis of the observed spectral patterns can reveal distinct spectral classes that correlate well with visual cytology [1]. The relevant part of the IR spectral region is generally taken to be 2-20 micrometers in wavelength. To date, most IR microspectroscopical studies have employed some version of diffraction-limited microscopy, of which one type provides optical visualisation plus IR spectroscopic data collection. The second (more recent and more advanced) technique using commercially available 2-D Fourier transform infrared (FTIR) microscopy imaging spectrometers with focal plane array (FPA) detection can produce images with pixels down to $5 \times 5$ $\mu \mathrm{m}$ in size with very good signal/noise ratio.

On the other hand, near-field photothermal versions can perform localised spectroscopy and imaging with a spatial resolution that is not diffraction-limited, which means that they achieve higher spatial resolution than is possible with conventional far-field optics [2]. These photothermal techniques are based on the fact that an object that absorbs IR radiation heats up. In the technique known as AFM-IR or photothermally-induced resonance (PTIR) [2-5], resonant motion of the probe induced by rapid expansion of the object is detected. Alternatively the temperature change can be detected directly by a temperature-sensitive probe, as PhotoThermal MicroSpectroscopy (PTMS) [6] which has advantages in a number of situations, in that often little or no sample preparation is required: as well as higher spatial resolution, it yields true absorption spectra even in situations where parts of the sample (for example, mitotic chromosomes) may be IR-opaque and thus give misleading data in the conventional transmission mode. In PTMS, imaging uses a scanning probe microscope (SPM) and a source of radiation focused onto the point where the probe contacts the surface of the sample. In each of the following examples, the relevant micro-anatomical components for analysis were identified using either transmission electron microscopy, confocal microscopy, phase-contrast microscopy or synchrotron FTIR microspectroscopy: where appropriate, paraffin-embedded tissue sections were stained with haematoxylin and eosin before being checked retrospectively by a pathologist.

Photothermal microspectroscopy (PTMS). In our first example of near-field microspectroscopical measurements, PTMS was employed, using the same type of thermal probe as is used in scanning thermal microscopy. This imaging probe is a sub-miniature temperature sensor, which may be a thermocouple or a resistance thermometer. As with a standard AFM, the probe is incorporated into a cantilever structure, so that force feedback may be employed in the usual way, for positioning and topographic imaging. The AFM is interfaced with an infrared spectrometer. A particular region of the sample may first be chosen on the basis of the image obtained using the AFM imaging mode of operation. The IR beam is focused to a spot, whose diameter is not critical. Then when material at this location absorbs the electromagnetic radiation, heat is generated which diffuses, giving rise to a decaying temperature profile. The thermal probe then detects the photothermal response of this region of the sample.

In one variant of PTMS, the light source is a bench-top tuneable laser in the form of an optical parametric amplifier (OPA) [5], tuned successively to different wavelengths. Alternatively, in common with the great majority of IR spectroscopic techniques, PTMS frequently employs a conventional globar broadband ("white") infrared source of radiation, giving a continuous spread of frequencies. With this broadband technique, an interferometer with a moving mirror allows the signal to be measured as a function of time (the "interferogram"). The data are then converted to intensity as a function of frequency (i.e. the spectrum) by means of the Fourier-transform method. In the conventional version, the measured signal is light intensity (e.g. transmitted or reflected). In broadband PTMS, the modulation of the light intensity by the interferometer causes modulation of the energy absorption by the sample. The diffusion of heat to the temperature sensor is thus modulated at the same frequency as the light modulation. The same principle as in conventional FTIR spectroscopy is used, except that the raw interferogram is simply the temperature measured by the probe as a function of time: the Fourier transform conversion then proceeds as usual.

These techniques have been used to characterise adult (non-embryonic) stem cells, both from the corneal epithelium and from the human gut. Identification of adult stem cell populations has proved 
very difficult, since by their very nature they appear undifferentiated: no established reliable markers for this purpose exist that are applicable across different anatomical sites, and to-day, the locations of many stem-cell populations in different tissues of the human body remain uncertain. Commonly there are three main types of cell: stem cells (SC), which can divide asymmetrically to give rise to another stem cell and a second cell type designated as a transit-amplifying (TA) cell; subsequent cell divisions of the TA cells will give rise to the third cell type given the designation terminally-differentiated (TD) cell (these are the functional cells of a specific tissue, though it must be recognised that there may be more than one type of TA cell in a micro-anatomical component).

\section{Data analysis issues}

The "needle in a haystack" problem. Next we turn to the question of data analysis. In a particular case we might, for instance, have two groups of tissue samples, with 50 samples in each group. Suppose that we wish to see whether the types of cell in the two groups differ significantly in the secondary structure of intracellular proteins, or the degree of protein phosphorylation. We measure the 100 spectra, each with 1000 wavebands, but it is very unlikely that any one single wavenumber in a spectrum will respond only to a particular alteration in either of these two factors. Indeed, there could be less than $1 \%$ difference in the amplitudes of the spectral peaks, between the various samples! To attack this "needle in a haystack" problem, one way forward is to find some way of ignoring the similarities and picking out the variability (mathematically, the differences in variance as derived from the correlation matrix of the data). This may be achieved by using chemometric methods involving multivariate data analysis [1], or alternatively non-statistical methods such as artificial neural networks [7] or fuzzy logic [8].

Principal component analysis. For example, in determining the observed variance pattern it would almost certainly be misleading to regard the hundreds of wavebands in the spectrum as independent variables. Methods such as principal component analysis (PCA) exploit the fact that instead, the variation is typically determined by perhaps only 4 or 5 factors ("principal components"). Each raw data spectrum presents data from a single sample, but of course involves all the wavenumber variables. With PCA, for each wavenumber variable we may tabulate, along a single axis, the value of that variable measured for each of the samples (Fig. 1). This gives us a single column vector.

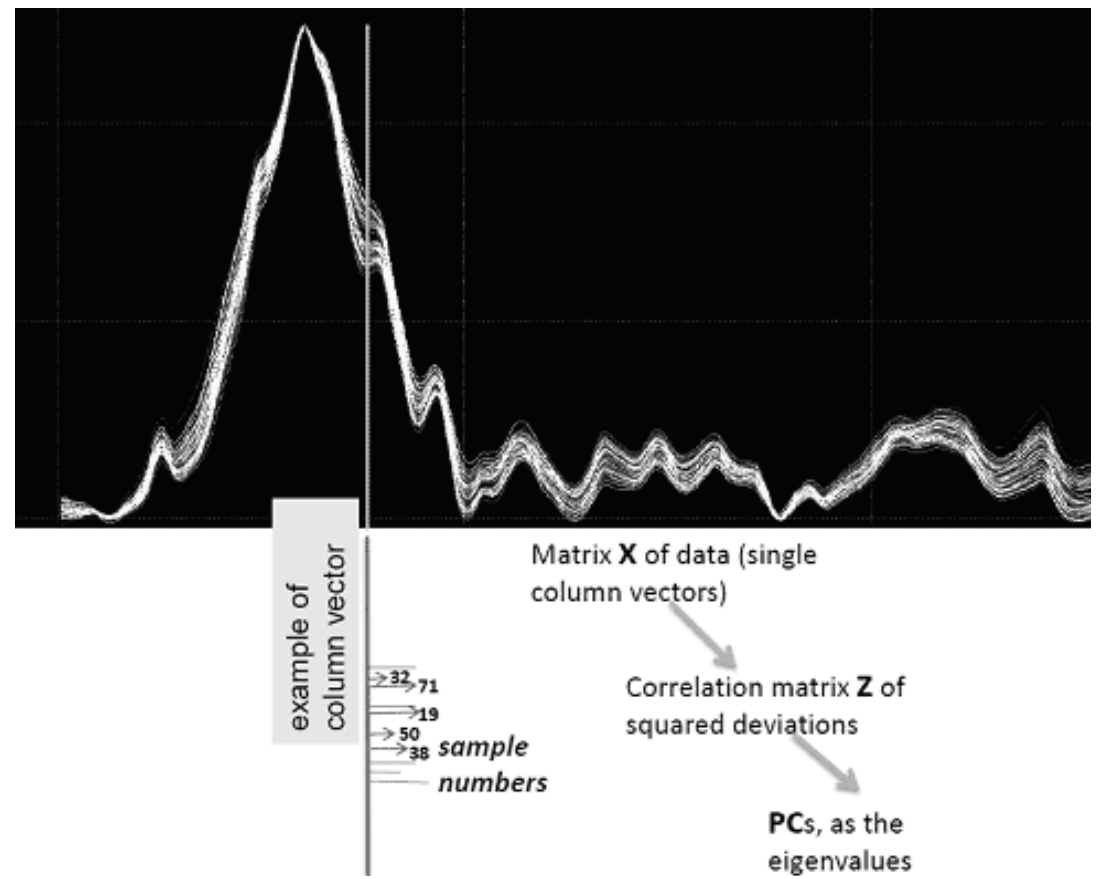

Figure 1: Example of a series of spectra, illustrating how principal components are derived (schematic) 
Software then uses matrix algebra to create a vector consisting of a linear combination of the many hundreds of these raw data vectors, calculated in such a way as to reveal maximal amounts of variation, which plotting the original vectors fails to enable us to achieve. Each of these vectors is called a principal component (PC), and these PCs act as axes in multidimensional "hyperspace", replacing the many hundreds of waveband variables (wavenumbers). The PCs appear in order, starting with PC1 which reveals the biggest spread between objects. The so-called "score" of each sample for a given PC is a measure of its contribution to the individual PC: plots of the scores of all the samples reveal intersample relationships (groupings or clusterings).

PCA is just one of a multitude of multivariate data analysis methods, each of which may have advantages according to the particular nature of the task. They are discussed in the review by Wang and Mizaikoff [1], and Fig. 12 of ref. [2] gives a chart that summarises the more popular of these.

Allocation of spectra from individual regions to different categories (clusters, groupings, classes). Our preference is use PCA for preliminary data reduction and to process the output using discriminant analysis (LDA), which is a "supervised" method in that a priori classes derived from PCA or from prior knowledge are pre-allocated. The spectra from one of the studies of corneal cells are shown as a PCA-LDA scores plot in Fig. 2, and we see that this PCA-LDA procedure [9] can be an extremely powerful method of revealing clustering.
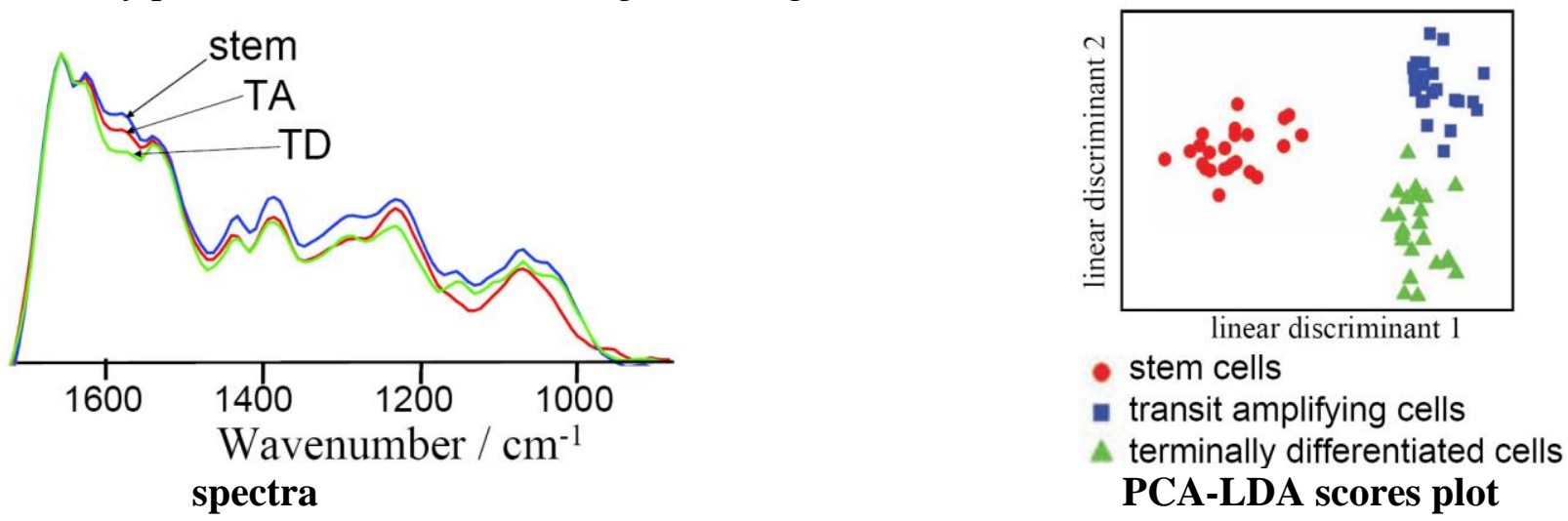

Figure 2: Identification of adult stem cell populations from the corneal epithelium. The PCA-LDA scores plot shows very clear separation of the different types of cell (O. Grude, A. Hammiche, H.M. Pollock, A.J. Bentley, M.J. Walsh, F.L. Martin and N.J. Fullwood, J. Microscopy Vol. 228, p. 366, (02007 Wiley-Blackwell, reproduced with permission).

Biomolecular features responsible for separation into classes? Just as a single raw data spectrum involves all the wavenumbers, the equivalent version of a $\mathrm{PC}$ vector, for plotting the contribution of each wavenumber to the PC, is termed its loadings curve: it presents data from all the samples, and plots the coefficients by which the original wavenumber variables must be multiplied to obtain the PC concerned. Thus it may be regarded as a pseudo-spectrum, but displaying variation rather than raw data. Accordingly, we may compare the original spectra with the loading curves of the PCs used, in order to identify the particular wavenumbers whose signal amplitudes reveal significant variation between samples. Thus once any groupings (clusters) have appeared, these critical wavenumbers provide a way of revealing the biomolecular and/or conformational changes that are associated with particular cell subpopulations, whether normal or otherwise.

A limitation of this use of PCA loadings is that unless one of the PCs happens to pass through the cluster in question, the vital wavebands responsible for the observed clustering might be missed. Thus, even more useful are the loading curves of individual "cluster vectors" or pseudo-PCs which, in a recent variation of this approach [9], may be plotted in hyperspace to pass though the medians of individual clusters of interest (Fig. 3). A weighted averaging algorithm then gives the required plot identifying the wavenumbers responsible. In the case of the corneal cells whose clustering is shown in Fig. 2, this method showed that the differences between the three cell types involved variations in the spectral peak heights as follows: 
$\mathrm{C}=\mathrm{O}$ stretching vibrations of nucleic acids $\left(1710-1700 \mathrm{~cm}^{-1}\right)$;

$\mathrm{C}=\mathrm{N}$ stretching in guanine $\left(1590 \mathrm{~cm}^{-}\right)$;

$\mathrm{COO}^{-}$symmetrical stretching vibrations of fatty acids and amino acids or $\mathrm{C}-\mathrm{H}$ bend in amino acid residues $\left(1400 \mathrm{~cm}^{-1}\right)$;

Phosphate activity $\left(1200-1190 \mathrm{~cm}^{-1}\right)$;

Sugar ring vibrations' and C-O and C-C stretch in DNA ${ }^{2}\left(1000-950 \mathrm{~cm}^{-1}\right)$

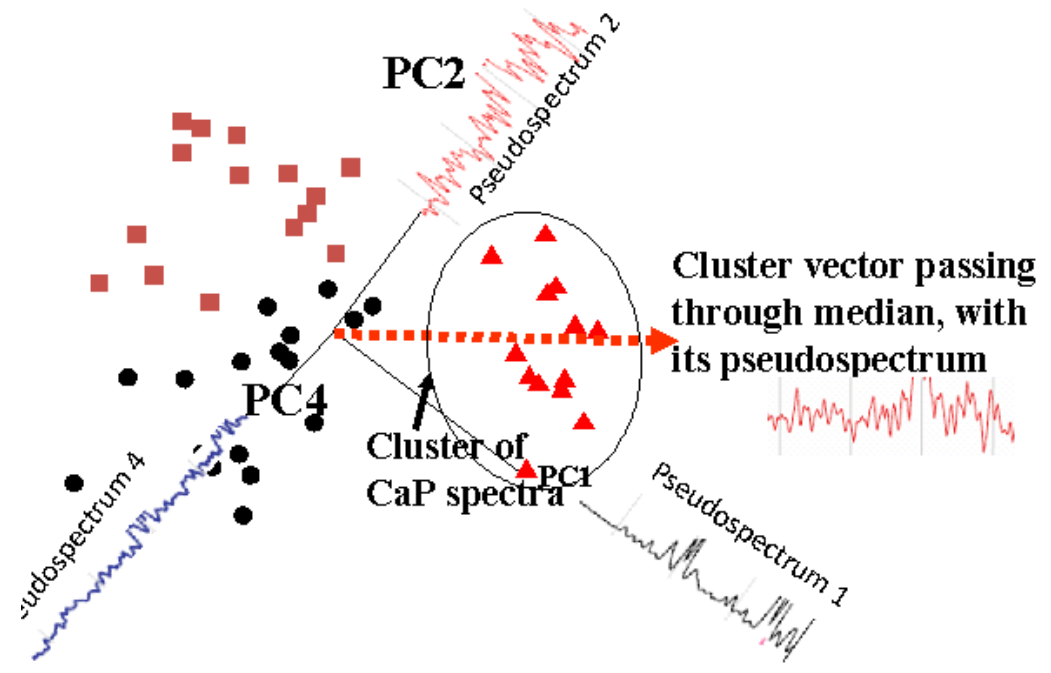

Figure 3: Scores plot with schematic representation of the loading curves ("pseudospectra") of three PCs, together with the resultant cluster vector constructed so as to pass though the median of one of the three clusters.

Differences versus average of all spectra:

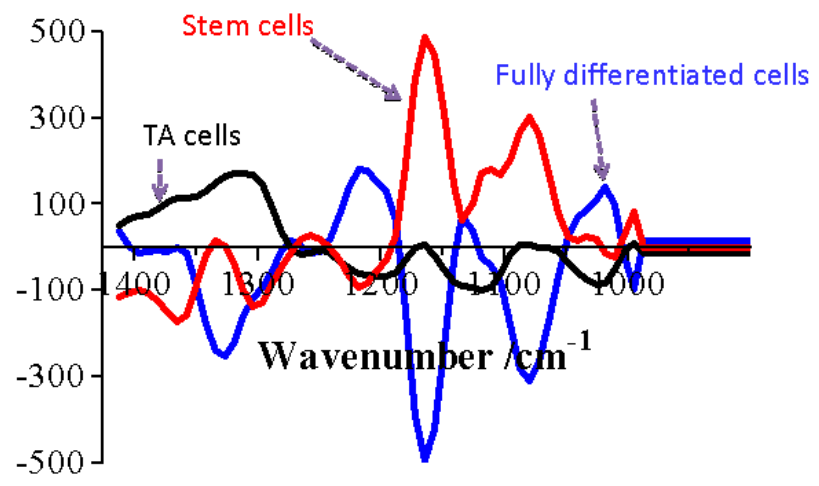

Figure 4: Loading curves showing which molecular groups were found to be responsible for the segregation into discrete data clusters of stem, TA and TD cells from crypts in the human large bowel:

protein conformation $\left(1800 \mathrm{~cm}^{-1}\right.$ to $\left.1480 \mathrm{~cm}^{-1}\right)$

asymmetric $\mathrm{PO}_{2}^{-}\left(1225 \mathrm{~cm}^{-1}\right)$ and $v_{\mathrm{s}} \mathrm{PO}_{2}^{-}\left(1080 \mathrm{~cm}^{-1}\right)$

symmetric $\mathrm{PO}_{2^{-}}$at $1080 \mathrm{~cm}^{-1}\left(1490 \mathrm{~cm}^{-1}\right.$ to $\left.1000 \mathrm{~cm}^{-1}\right)$,

which is consistent with reports of stem cell differentiation being triggered by chromatin modifications. Each loading curve is the pseudospectrum of the hyperspace vector passing through the median of the relevant scores cluster, and compares that type of cell's PTMS spectrum with the average spectrum obtained from all classes. The samples were thin sections, obtained from paraffinembedded tissue blocks and mounted on reflective glass slides (M.J. Walsh, T.G. Fellous, A. Hammiche, W-R. Lin, N.J. Fullwood, O. Grude, F. Bahrami, J.M. Nicholson, M. Cotte, J. Susini, H.M. Pollock, M. Brittan, P.L. Martin-Hirsch, M.R. Alison and F.L. Martin, Stem Cells Vol. 26, p. 108, (C) AlphaMed Press 2008, reproduced with permission). 
In Fig. 4 we show an example of loadings curves taken from a recent paper [10] explaining how the location of the putative stem cell location may be discriminated from that of TA and TD cell locations, in another type of adult human stem cell found in intestinal crypts (the regular deep infoldings of intestinal epithelium which incorporate a proliferating compartment giving rise to replacement cells for shed surface epithelium) in both small and large bowel.

Further examples of the use of near-field photothermal micro-spectroscopy with data analysis using PCA-LDA in biomedical research may be found in [2, 10-16]. In a study of exfoliative cervical cytology [15], it was found that biomarkers of progression from normal to lowgrade, high-grade and severe dyskaryosis included the degree of protein phosphorylation at a wavenumber of $970 \mathrm{~cm}^{-1}$, glycogen at $1030 \mathrm{~cm}^{-1}$, and shifts in the centroid of the amide I peak at around $1650 \mathrm{~cm}^{-1}$, indicating changes in the secondary structure of intracellular proteins. Cancer of the prostate gland, which is composed of a number of different zones (peripheral, transitional, and central), has also been studied using microspectroscopy [12]. Many of the spectra from epithelial cells in the transitional zone, as compared with those from normal cells, show significant biochemical alterations as indicated by ratios of peak heights (e.g. RNA vs. DNA, carbohydrate vs. phosphate). Such differences may correlate with genetic changes, possibly indicating cancerinitiating features. Indeed, these TZ spectra have much in common with spectra from cancerous cells. However, most of the pathology in this zone is benign despite the occurrence of this cancerlike phenotype, which in the absence of a suitable growth-promoting stimulus remains nonmalignant. To add to this paradox, the spectra from cancerous cells do not resemble most of the spectra from the peripheral zone, where most carcinoma occurs. This suggests that for individual cells, what is crucial is not their degree of susceptibility to the initiating events associated with mutation hits. Instead, probably the most important factor is that in the peripheral zone, there is a much larger number of epithelial cells which (despite their lower mutation-susceptibility), once they have mutated, can act as targets for stimuli such as hormone exposure that may produce malignant growth.

\section{A non-statistical method of classification, employing fuzzy logic}

The photothermally-induced resonance technique. In this second example of near-field spectroscopic characterization of biomaterials, instead of PTMS the AFM-IR (PTIR) technique was used [17]. Here, this photothermally-induced resonance method of detection employed top-down illumination from a bench-top OPA. The OPA pulse produces a very rapid transient local absorption-induced heating of the sample, and this is detected via the transient resonances of the cantilever vibration [3-5]. The fast Fourier transform (FFT) power density spectra of the cantilever deflection as a function of wavelength are then recorded. Using such data, it is possible to group the different samples into classes: in addition, we may perform infrared mapping, where the confidences of classification are used to determine image contrast. In this experiment, FFT spectra were obtained at ten different probe positions on a polystyrene sample in the form of a $100-\mu \mathrm{m}$ bead supported on mica as represented in Fig. 5, where positions 1-5 and 6-10 are on-sample and offsample locations respectively.

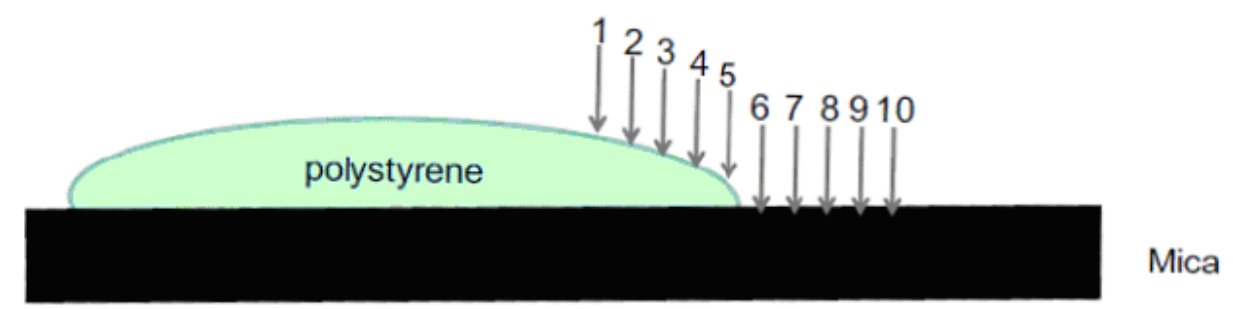

Figure 5: Polystyrene sample, showing on-sample (1-5) and off-sample (6-10) locations at which photothermally-induced resonance FFT spectra were obtained 
We present this example as an illustration of possible advantages of employing a non-statistical method of data analysis such as "eClas", a self-learning classifier that employs fuzzy logic $[8,18]$. eClas is a supervised method, and training is performed using a set of data of known class. After validation of the model, it is used to test the remaining data. The output gives a reading of class (e.g. on-sample versus off-sample) for each set of data, together with a value of confidence of assignment to its class which can also provide the basis of a method for generating image contrast. An evolving Tagaki-Sugeno type of fuzzy rule-based model such as eClas addresses the challenge of how to interpret data in real time. The model structure can evolve gradually, according to the changing requirements of a data stream. Each retraining iteration will modify only one, or a few, of the fuzzy rules. This gives the fuzzy approach an advantage over some alternatives, where iterative retraining could lead to a loss of previous information.

Determination of classification confidence and IR mapping. Fig. 6 shows that the resulting classification is consistent with the known distribution of the two materials:

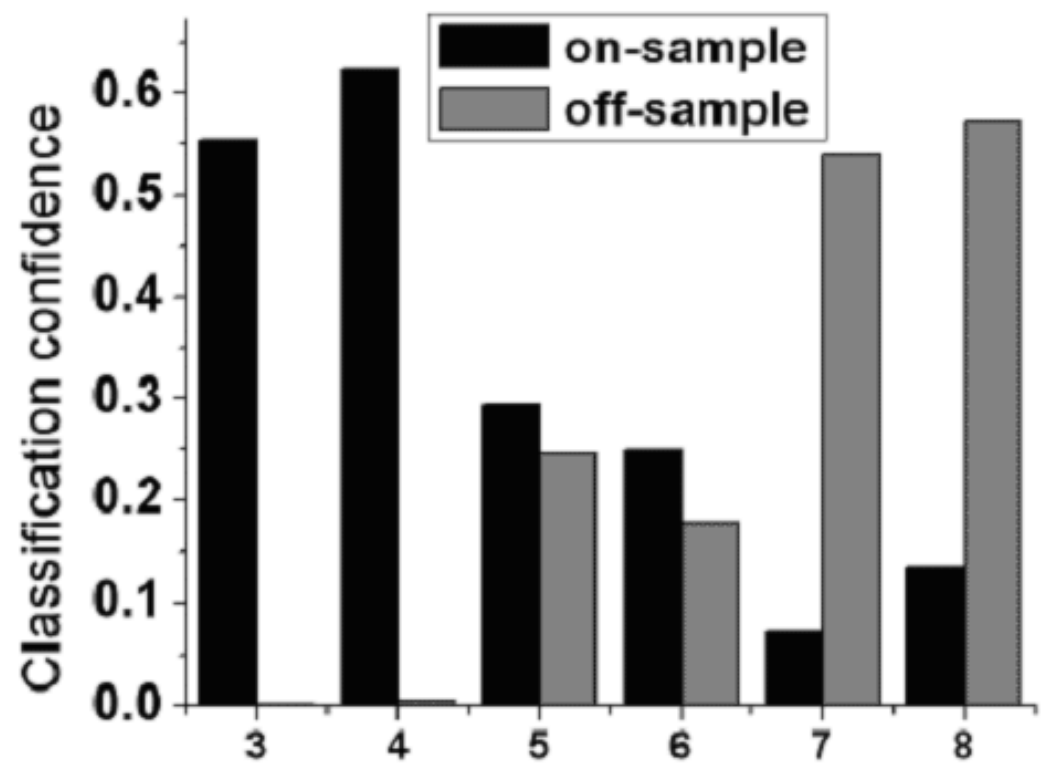

Figure 6: Confidences of classification (on- or off-sample), derived from the FFT data recorded at the locations shown in Fig. 5. Data from the extreme locations 1,2, 9 and 10 were used for training, and the figure shows classification results for the intermediate locations. The signal to noise ratio is excellent, as may be seen by comparing each bar with the sample location as indicated in the diagram.

Of course this method may be applied to PTMS data also: in another experiment [19], a twocomponent sample was prepared, in the form of a microtomed section having a sharp boundary between Araldite resin and polyethylene terephthalate (PET). A topographic image showed this border running vertically down the image, and PTMS spectra were acquired in steps of $0.5 \mu \mathrm{m}$ in horizontal lines from one material through the border to the other material. The sequence of spectral acquisition and probe movement was carried out many times, until an array of spectra measuring 1 by $30 \mu \mathrm{m}$ was created with a spatial resolution of $0.5 \mu \mathrm{m}$. Training spectra having known a priori classes were chosen from the edge regions of the sample, assumed to be pure PET and Araldite. After satisfactory validation, the model was used for testing all the spectra in the central region of the sample spanning the boundary between the two materials. Classification confidences ranged from $100 \%$ down to zero in the centre, as shown in Fig. 7. This may also be shown clearly on an IR map, where confidence of assignment to the class as determined by the fuzzy rule-based model may be plotted to give an "image" (see Fig. 8). The PET/Araldite boundary shows up in these classification confidence data, as a vertical low- confidence gap where the spectra were characteristic of neither PET nor Araldite. 


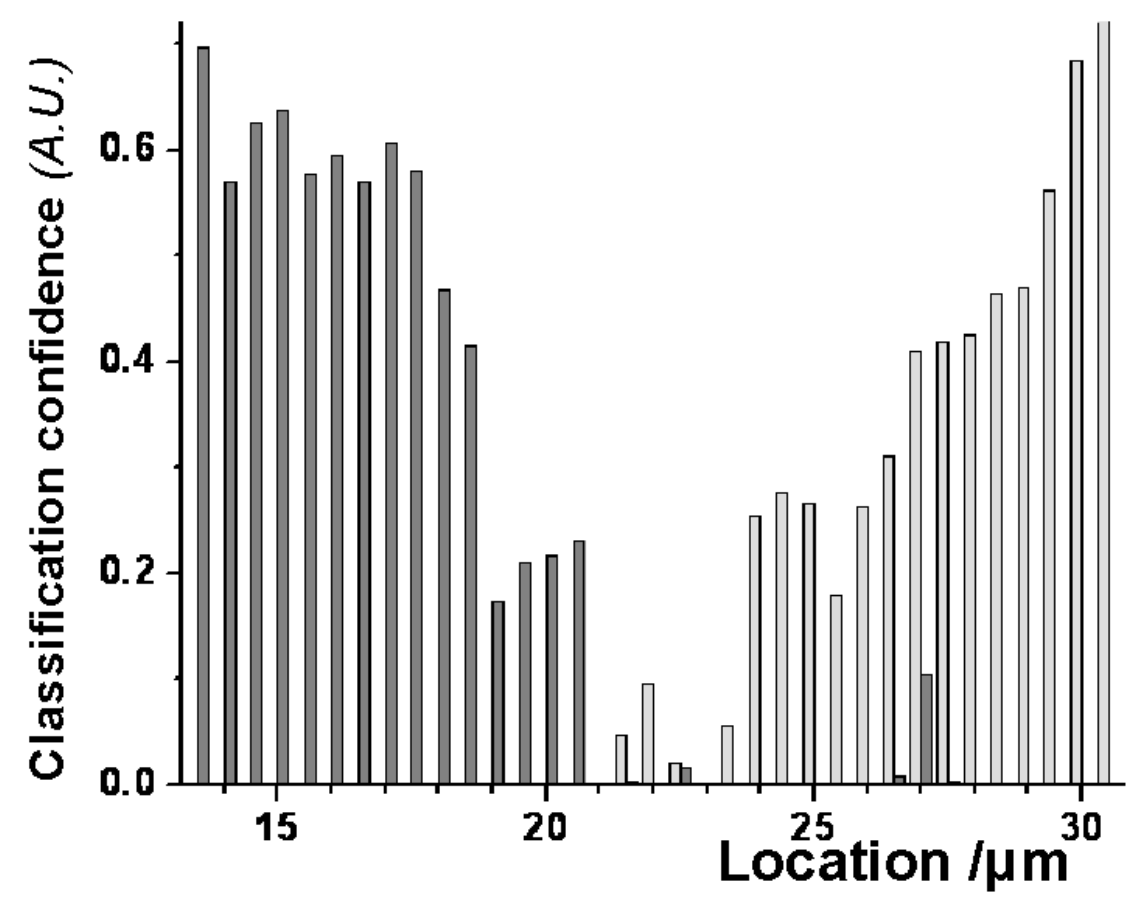

Figure 7: Classification confidences derived from PTMS data obtained at locations straddling an Araldite/PET boundary (x-scale in microns)

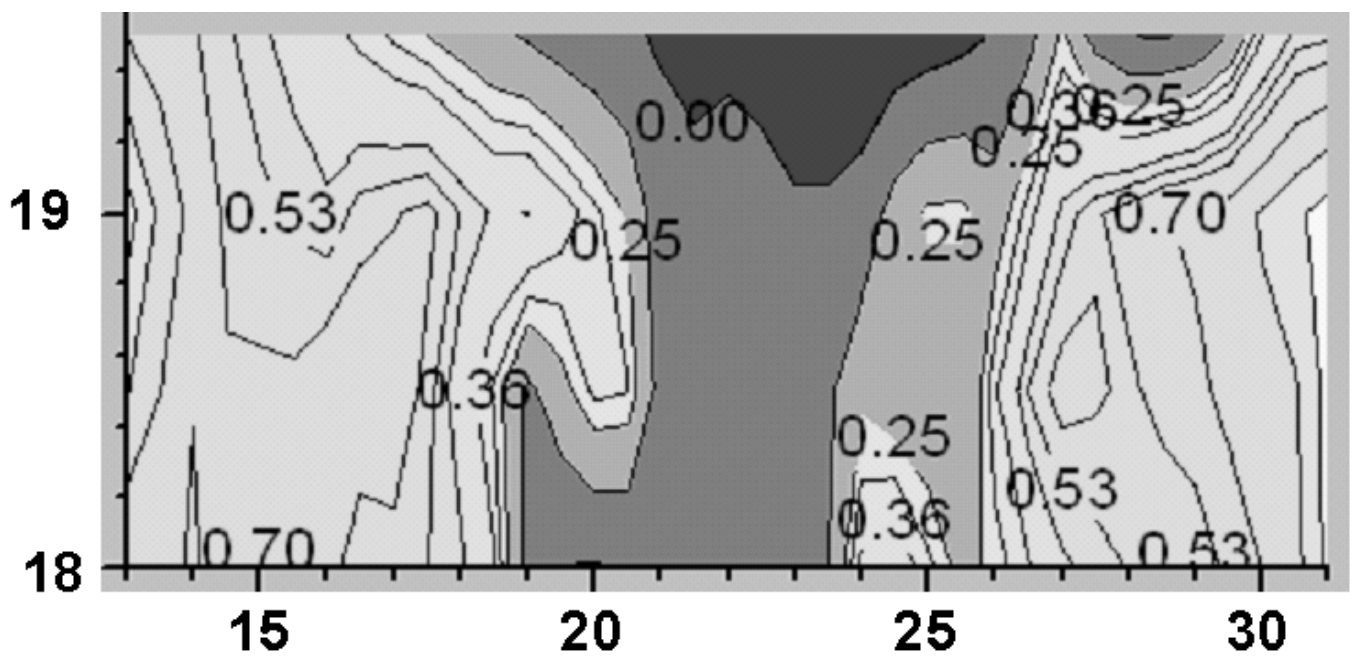

Figure 8: Example of an IR map derived from classification confidences, using PTMS data obtained at 80 locations on an Araldite/PET sample. The boundary between the two materials runs vertically up the centre of the image. (In this figure, the numerical values and false greyscale intensities shown refer to Araldite on the left and PET on the right)

\section{Molecular groups primarily responsible for delineation}

The training stage yields an additional type of information, called "feature weights": the fuzzy rules are the key component of the model that is being determined during training, which reveals which features are primarily responsible for the separation into classes. It is possible to tabulate the relative importance of each feature (e.g. FFT frequency, or each wavenumber, depending on the nature of the data), as measured by its "degree of accumulated fuzzily-weighted usage" in helping to determine the fuzzy rules.

A graph of these weights as a function of wavenumber thus provides an interesting alternative to a loading curve derived from the same data by PCA-LDA, in order to determine which molecular groups are primarily responsible for the separation into classes. A comparison of these very different approaches is described elsewhere [19]. 


\section{Possible applications of the fuzzy logic approach}

Given these initial results on Araldite-based samples, these latter approaches should in principle be applicable to resin-embedded biological sections. It should, therefore, be possible to match IR maps of tissue sections to images obtained by conventional transmission techniques, and thus correlate any non-random patterns identifiable with the known micro-anatomical profile.

\section{Concluding remarks: could the use of spectroscopic biomarkers become part of biomedical evaluation procedures?}

Given such methods of data analysis and classification, there is no fundamental obstacle to further improvements to these PTMS and PTIR techniques to the point where further challenges can be met: for example, routine imaging of subcellular detail, the detection of significant inter-class differences in the base structure of cellular DNA, etc., provided that the necessary advances in probe fabrication and other instrumental technology can be developed. An exciting achievement would be a single cell map where different molecular components within the single cell would be clearly correlated with specific well-recognised subcellular compartments. Of particular interest would be the possibility of recognising meaningful variation in nuclear substructure that may correlate with function and differentiation. This is not possible with commercial micro-FTIR, and would greatly enhance the impact of microspectroscopy for biomedical scientists.

In circumstances where significant diagnostic difficulties arise in the assessment of recurring relatively common problems, the favoured type of evaluation is known as histopathological consensus diagnosis, where several histopathologists deliver an opinion on the same sample set. To satisfy the current gold standard represented by established procedures is time-consuming and expensive, requires an appropriate number of suitably-qualified experts, and remains open to unresolved differences of opinion. A major disadvantage of microscopic methodologies such as confocal microscopy is that in general, they are restricted to the mapping, in any one experiment, of only one or a few biomarkers which may be identified through the application of an antibody or other specific binding molecule suitably labelled with a fluorescent tag. Moreover a lung biopsy, for example, may contain only a small number of suspicious cells, sometimes insufficient in number for a diagnosis of cancer to be agreed. The use of vibrational spectra thus provides the tempting alternative possibility of providing a diagnosis from a very small number of cells, since spectral absorbance bands form strong candidates to provide robust biomarkers: examples include amide I $(\approx 1650 \mathrm{~cm}-1)$, asymmetric phosphate $\left(v_{\mathrm{as}} \mathrm{PO}_{2}^{-}, \approx 1225 \mathrm{~cm}-1\right)$; symmetric phosphate $\left(v_{\mathrm{s}} \mathrm{PO}_{2}^{-} \approx 1080\right.$ $\mathrm{cm}-1)$; and glycogen $(\approx 1030 \mathrm{~cm}-1)$. Refs. [8] and [15] discuss the example of cervical cytology, where although there already exist semi-automated morphometric and related techniques which, in principle, can identify suspect cells or clusters, the possibility of developing a fully quantitative and objective procedure is a realistic target.

However, establishing such a major change in procedures would be a formidable task. Relatively small spectral changes are generated that are heavily dependent on chance spatial variation in which part of the sample is to be analysed. Moreover, complications arise from the different ratios and/or conformations of biomolecules that give rise to subtle changes in absorbance peaks (i.e. shape, shift and/or intensity) and indicate intracellular alterations. If this approach is to bear fruit, the establishment of a new diagnostic gold standard, together with appropriate information-processing algorithms, will be essential. Existing modalities which permit analysis of progression of a disease (or progression from stem cell to differentiated cell) must be used to validate any proposed evaluation procedure [2]. 


\section{Acknowledgments}

I gratefully acknowledge the experimental and interpretative work performed by colleagues at the University of Lancaster (UL) and the University of East Anglia (UEA), within the following research fields in particular: (PTMS) Azzedine Hammiche (UL) and Mike Reading (UEA); (stem cells) Nigel Fullwood (UL), Matt German (UL), Olaug Grude (UL) and Frank Martin (UL); (PTIR and polymer science) Jonathan Moffatt (UEA) and Mike Reading (UEA); and (fuzzy classification) Plamen Angelov (UL), Olaug Grude (UL) and Jemma Kelly (UL). Certain passages of biomedical relevance have been revised in accordance with constructive criticism made by one of the referees in particular, whom I thank accordingly.

\section{References}

[1] L. Wang and B. Mizaikoff, Anal. Bioanal. Chem. Vol. 391 (2008), p. 1641

[2] F.L. Martin and H.M. Pollock, in: The Oxford handbook of nanoscience and technology, volume II (materials: structures, properties and characterization techniques), edited by A.V. Narlikar and Y.Y. Fu, pp. 285-336, Oxford University Press, Oxford, England (2010)

[3] A. Dazzi, R. Praveres, F. Glotin and J.M. Ortega, Optics Letters Vol. 30 (2005), p. 2388

[4] A. Dazzi, R. Praveres and F. Glotin, Infrared Physics and Technology Vol. 49 (2006), p. 113

[5] G.A. Hill, J.H. Rice, S.R. Meech, D.Q.M. Craig, P. Kuo, K. Vodopyanov and M. Reading, Optics Letters Vol. 34 (2009), p. 431

[6] A. Hammiche, L. Bozec, M.J. German, J.M. Chalmers, N.J. Everall, G. Poulter, M. Reading, D.B. Grandy, F.L. Martin and H.M. Pollock, Spectroscopy Vol. 19(2) (Feb. 2004), p. 20 with erratum in Vol. 19(5) (2004) p. 14

[7] M. Wenning, S. Scherer and D. Naumann, in: Vibrational Spectroscopy for Medical Diagnosis, edited by M. Diem, J.M. Chalmers and P.R. Griffiths, Wiley, Chichester, England (2008).

[8] J.G. Kelly, P. Angelov, M.J. Walsh, H.M. Pollock, M.A. Pitt, P.L. Martin-Hirsch and F. L. Martin, Int. J. Computational Intelligence Research Vol. 4 (2009), p. 392

[9] F.L. Martin, M.J. German, E. Wit, T. Fearn, N. Ragavan and H.M. Pollock, J Computational Biology Vol. 14 (2007), p. 1176

[10] M.J. Walsh, T.G. Fellous, A. Hammiche, W-R. Lin, N.J. Fullwood, O. Grude, F. Bahrami, J.M. Nicholson, M. Cotte, J. Susini, H.M. Pollock, M. Brittan, P.L. Martin-Hirsch, M.R. Alison and F.L. Martin, Stem Cells Vol. 26 (2008), p. 108

[11] M.J. German, H.M. Pollock, B. Zhao, F.L. Martin, M. Tobin, A. Hammiche, A. Bentley, L.J. Cooper, F.L. Martin and N.J. Fullwood, Investigative Ophthalmology \& Visual Science Vol. 47 (2006), p. 2417

[12] M.J. German, A. Hammiche, N. Ragavan, M.J. Tobin, L.J. Cooper, N.J. Fullwood, S.S. Matenhelia, A.C. Hindley, C.M. Nicholson, N.J. Fullwood, H.M. Pollock and F.L. Martin, Biophysical Journal Vol. 90 (2006), p. 3783, with supplementary data

[13] O. Grude, A. Hammiche, H.M. Pollock, A.J. Bentley, M.J. Walsh, F.L. Martin and N.J. Fullwood, J Microscopy Vol. 228 (2007), p. 366

[14] A.J. Bentley, T. Nakamura, A. Hammiche, H.M. Pollock, F.L. Martin, S. Kinoshita and N.J. Fullwood, Molecular Vision Vol. 13 (2007), p. 237 
[15] M.J. Walsh, M.N. Singh, H.F. Stringfellow, H.M. Pollock, A. Hammiche, O. Grude, N.J. Fullwood, M.A. Pitt, P.L. Martin-Hirsch and F.L. Martin, Biomarker insights Vol. 3 (2008), p. 179

[16] O. Grude, T. Nakamura, A. Hammiche, A.J. Bentley, F.L Martin, H.M. Pollock, S. Kinoshita and N.J. Fullwood, Vibrational Spectroscopy Vol. 49 (2009), p. 22

[17] J.G. Moffat, X. Dai, J. Wood, P.S. Belton, P.Angelov, H.M. Pollock and M. Reading (2010a), in preparation.

[18] P. Angelov and X. Zhou, in: Proc 2006 Int Conf Evolving Fuzzy Systems, Ambleside, England (7-9 Sept 2006), IEEE Press.

[19] J.G. Moffat, X. Dai, J. Wood, P.S. Belton, P.Angelov, H.M. Pollock and M. Reading (2010b), in preparation. 